\title{
Acceso a materiales de estudio universitarios en Uruguay
}

\author{
Virginia Rodés ${ }^{1,3}$, Patricia Díaz ${ }^{2,3}$, Jorge Gemetto ${ }^{3}$, Mariana Fossatti ${ }^{3}$ \\ 'Programa de Entornos Virtuales de Aprendizaje, Comisión Sectorial de Enseñanza \\ Universidad de la República (Udelar) \\ José E. Rodó 1854 - C.P. 11200- Montevideo - Uruguay \\ ${ }^{2}$ Instituto de Computación, Facultad de Ingeniería, Universidad de la República (Udelar) \\ Julio Herrera y Reissig 565 - C.P. 11.300 - Montevideo - Uruguay \\ ${ }^{3}$ Creative Commons Uruguay \\ Eduardo V. Haedo 2255 - CP 11200 - Montevideo - Uruguay \\ vrodes@cse.edu.uy, pdiazefing.edu.uy, jorgemetegmail.com, \\ mfossatti@gmail.com
}

\begin{abstract}
While the term access is often associated with enrollment, access to higher education includes not only the time in which the student enrolls in college. Among many other factors, access to study materials becomes relevant when entering and staying in the education system. In the article a state of the art and the main debates about access to study materials in Uruguay are introduced, in order to know the current situation and guide educational policies, especially those related to Open Educational Resources as institucional policy publication of educational content.
\end{abstract}

Resumen. Si bien el término acceso suele asociarse a la matriculación, el acceso a la Educación Superior no solo incluye el momento en el cual el estudiante se matricula en la universidad. Entre otros múltiples factores, el acceso a los materiales de estudio pasa a ser un punto relevante al momento de ingresar y permanecer en el sistema educativo. En el artículo se presenta el estado del arte y los principales debates sobre el acceso a los materiales de estudio en Uruguay, de modo de conocer la situación actual y orientar politicas educativas, en especial aquellas vinculadas al desarrollo de los Recursos Educativos Abiertos como política institucional de publicación de contenidos educativos.

\section{Introducción}

En 2011, casi 1 millón de personas estaba dentro del sistema educativo en Uruguay. Entre ellas, unas $330 \mathrm{mil}$ en el nivel medio, casi $30 \mathrm{mil}$ en el nivel terciario no universitario y poco más de 100 mil en el nivel universitario, según INE (2013). Prácticamente el 100\% de la educación terciaria no universitaria en Uruguay es pública.

En cuanto a la educación universitaria, aproximadamente el 85\% de los estudiantes universitarios asiste a la Universidad de la República (Udelar), la cual es pública. Según el Censo de Estudiantes Universitarios de grado de la Udelar (Udelar, 2012), en 2012 había 85.905 estudiantes solo en carreras de grado. El resto de los 


\section{CBIE-LACLO 2015}

Anais dos Workshops do IV Congresso Brasileiro de Informática na Educação (CBIE 2015)

estudiantes universitarios se distribuye entre poco más de 10 universidades e institutos universitarios privados.

Estas características del escenario de la Educación Superior en Uruguay ubican a la Udelar como la gran protagonista en la formulación de políticas para la generalización del acceso a la Educación Superior.

La Educación Superior es un Derecho Humano, tal como lo expresa la Declaración de la Conferencia Regional de Educación Superior en América Latina y el Caribe (IESALC, 2008), donde se sostiene que "La Educación Superior es un derecho humano y un bien público social. Los Estados tienen el deber fundamental de garantizar este derecho (...) velando por que ella sea pertinente y de calidad". La Educación como Derecho Humano genera responsabilidad a los Estados de asegurar el acceso generalizado a la misma a través del diseño de políticas que garanticen equidad.

Si bien el término acceso suele asociarse a la matriculación, el acceso a la Educación Superior no solo incluye el momento en el cual el estudiante se matricula en la universidad. Como afirma Dias Sobrinho (2008), “[...] el acceso a la educación superior no debe ser considerado solamente como el "momento de entrada" del estudiante a la IES, sino como un proceso que se inicia en los niveles primarios y medios de educación, se extiende con la llegada a la institución docente y se enlaza con la permanencia en el programa de estudios. Por tanto, las políticas públicas deben tomar en consideración que, en relación a los estudiantes de educación superior, no se trata solamente de acceder, sino de mantenerse estudiando, graduarse y lograr empleos adecuados y coherentes con su formación; esto va a permitir a las personas ser ciudadanos más responsables, tener las oportunidades para disfrutar de una vida plena y poder ejercer la totalidad de sus derechos sociales".

Entre otros múltiples factores, el acceso a los materiales de estudio pasa a ser un punto relevante al momento de ingresar y permanecer en el sistema educativo.

Son los estudiantes de menores recursos los más afectados por el gasto en materiales educativos con derechos de autor en el marco de la formación universitaria, como puede observarse, por ejemplo, en un estudio llevado adelante en San Pablo, Brasil, por Craveiro, Machado y Ortellado (2008). Según el citado estudio, para el $90 \%$ de los estudiantes consultados en la investigación, los costos anuales de la compra de libros eran cercanos, o incluso superan, a la renta familiar mensual. Las bibliotecas no estarían en condiciones presupuestales de cubrir esta distancia tan grande entre los presupuestos familiares y las necesidades de los estudiantes. Además, el estudio descubrió que un tercio de los libros en la base de datos bibliográfica estudiada estaban agotados, es decir, no disponibles en el mercado. Adicionalmente, pone en evidencia que la producción de libros de texto, incluyendo la elaboración de contenidos (vía sueldos y becas a docentes e investigadores de las propias universidades) y la edición (en editoriales asociadas muchas veces a las universidades), es financiada mayormente por fondos públicos, siendo la inversión pública al menos 9 veces mayor que la privada.

Ante la falta de consideración del tema desde la perspectiva de los Derechos Humanos han surgido varios movimientos que bregan por el principio de acceso al conocimiento científico. Entre ellos podemos citar al movimiento por el Acceso Abierto a la Investigación Científica (Open Access) (BOAI, 2002) y el movimiento A2K (Access to Knowledge) (Noronha y Malcolm, 2010). 
En lo que refiere específicamente al tema de acceso a los materiales de estudio, encontramos al movimiento por los Recursos Educativos Abiertos (REA u OER por su sigla en inglés) que impulsa políticas para disponibilizar y descentralizar la publicación de recursos educativos, así como las herramientas para su creación. Èste movimiento surge en el mundo académico a partir de la iniciativa de liberación de los cursos (OpenCourseWare) del Instituto de Tecnología de Massachussets (MIT) en el años 2001. En el 2005 el Instituto Internacional para la Planificación Educativa de la UNESCO (IIEP) lanzó un foro de discusión sobre REA afirmando sus principios y dándole carácter global al movimiento. Y más recientemente, en el año 2012, éste mismo foro de la UNESCO, a través de la Declaración de París, exhorta a los gobiernos de todo el mundo al licenciamiento abierto de los materiales educativos de uso público financiados por el Estado.

En base a estos antecedentes y consideraciones es que utilizaremos el concepto de ecosistema para identificar un modelo de creación, circulación y acceso a los materiales de estudio, tanto en modalidad física como digital. En la Sección 2 del artículo se hace foco en los debates recientes que se han dado en el país en esta materia. La Sección 3 describe la situación actual del ecosistema de materiales de estudio en Uruguay. Finalmente, en la Sección 4 se establecen algunas conclusiones y líneas de trabajo futuras a efectos de orientar políticas educativas, en especial aquellas vinculadas al desarrollo de los Recursos Educativos Abiertos como política institucional de publicación de contenidos educativos.

\section{Debates recientes en Uruguay sobre el acceso a materiales de estudio y derechos de autor}

Desde el año 2013 han tenido lugar discusiones profundas sobre la tensión entre el derecho de autor y el acceso al conocimiento en Uruguay. La discusión pública se inició en el mes de julio de 2013, cuando el Ministerio de Educación y Cultura elevó al Parlamento una propuesta para aumentar el plazo de derecho de autor en 20 años, hasta los 70 años post-mortem. Esta propuesta generó una intensa polémica y provocó la conformación de un movimiento social de jóvenes, artistas, estudiantes e intelectuales con el objetivo de frenar dicha medida por considerar que la misma atentaba contra el derecho de acceso a la cultura y el conocimiento (Gemetto, 2013).

A raíz de las protestas, el Ministerio de Educación y Cultura (MEC) retiró dicha propuesta y se comprometió a promover la discusión ciudadana sobre el tema. A comienzos de noviembre de 2013, organizó las conferencias ciudadanas sobre Derechos de Autor y Acceso a la Cultura (Conferencias SUMAR). Como resultado de las conferencias, quedó a disposición de la ciudadanía una gran cantidad de materiales en formato escrito y audiovisual (Sumar Uy, 2013) sobre este tema.

Durante el mes de octubre de 2013 tuvo lugar un operativo policial de grandes proporciones, donde se allanó numerosos locales de fotocopiado en las cercanías de establecimientos universitarios y en particular frente a la Facultad de Derecho de la UdelaR. La Federación de Estudiantes Universitarios emitió una declaración en favor del acceso libre al conocimiento (FEUU, 2013) y, días más tarde, tuvo lugar una manifestación en favor de una reforma de la ley de derecho de autor, con un acto en la vía pública y la presentación de más de 10.000 firmas de apoyo (El País, 2013). A mediados de 2014 se presentó en el Parlamento un proyecto de ley para facilitar el 
acceso a materiales de estudio mediante excepciones y limitaciones al derecho de autor. Al día de la fecha dicho proyecto se encuentra a estudio del Parlamento.

Por último, tanto a nivel del MEC como de la Udelar, se ha iniciado la búsqueda de soluciones y comenzaron a funcionar comisiones que analizan el tema del acceso a los materiales de estudio. Estas comisiones han propuesto el desarrollo de los Recursos Educativos Abiertos y el Acceso Abierto como parte de la solución a los problemas del acceso.

Otros organismos públicos también han presentado propuestas. Por ejemplo, la Dirección Nacional de Impresiones y Publicaciones Oficiales (IMPO) implementó una librería digital y desde el Plan Ceibal (el plan 1a1 OLPC de Uruguay) se plantea la entrega de tablets a los estudiantes para facilitar la lectura en formatos digitales. Cada una de estas propuestas conlleva modelos diferentes en relación al modo de disponibilizar y abrir los materiales de estudio. Por ejemplo, la posibilidad o no de impresión y/o copia; el tipo de software, privativo o libre, utilizado para su desarrollo; y el grado de apertura de los materiales, entre otros factores, son diferentes en cada propuesta.

La crisis en el acceso a los materiales de estudio generada a partir de los allanamientos a locales de fotocopiado en 2013 evidencia, por un lado, que existe una brecha entre las prácticas sociales y la actual normativa, y por otro, la necesidad de que el Estado asuma un rol activo a los efectos de promover una coexistencia adecuada entre los derechos a la educación y la cultura, y los derechos de propiedad intelectual.

En este marco, podemos preguntarnos, ¿cuál es la situación en torno al acceso al conocimiento y los materiales de estudio en Uruguay? y ¿cuánto sabemos acerca de la forma en que los estudiantes y docentes universitarios producen y acceden a estos materiales de estudio en la práctica?

\section{Situación actual del ecosistema de materiales de estudio universitarios}

No existen datos exhaustivos respecto a la forma exacta en que los estudiantes universitarios en Uruguay, ni en particular en la UdelaR, acceden a los materiales de estudio. Tampoco existen estudios que describan el ecosistema de producción, circulación y acceso de material bibliográfico, definido este último como el sector de libros universitarios: académicos y técnicos. Por lo que efectuaremos una aproximación al tema de acuerdo a los antecedentes disponibles.

El concepto de ecosistema ha comenzado a ser utilizado para describir ambientes de producción, reutilización, uso y/o adaptación de contenido (Chang y West, 2006). Con el uso de este concepto se busca trascender los entornos de producción y uso tradicionales, rigurosamente definidos y centralizados, para favorecer la identificación de modelos híbridos, flexibles, abiertos y basados en la demanda (Boley y Chang, 2007).

Podemos afirmar que un ecosistema de producción y acceso a materiales educativos está conformado por distintos actores (estudiantes, docentes, autores, autores/docentes, editoriales nacionales y extranjeras, distribuidoras, librerías, bibliotecas, bibliotecas digitales, repositorios online, Instituciones Educativas, Entornos Virtuales de Aprendizaje y el propio Estado) interactuando entre sí, en un entorno tecnológico, económico, jurídico, político y social cambiante. 


\section{CBIE-LACLO 2015}

Anais dos Workshops do IV Congresso Brasileiro de Informática na Educação (CBIE 2015)

Entre estos actores circulan flujos de intercambio de conocimiento y recursos. El ecosistema tradicional es aquel en el que editoriales, distribuidoras y librerías controlan las cadenas de intercambio y retienen la mayor parte del valor como intermediarios entre el autor y el estudiante. Podemos decir que este ecosistema se mantiene en equilibrio gracias a las publicaciones estudiantiles, las bibliotecas universitarias y la comercialización y canje de libros usados. Este ecosistema tradicional funciona correctamente en un contexto de Universidad no masificada y bibliotecas con presupuestos adecuados a la demanda.

En las últimas décadas, el modelo planteado por el ecosistema tradicional se encuentra en crisis en Uruguay, el entorno se modifica, la educación superior se masifica cada vez más ${ }^{1}$ e irrumpen nuevas tecnologías que cambian las relaciones y alteran el equilibrio, como las fotocopias e Internet. Las editoriales de libros de texto observan cómo un mercado masificado en el que podrían expandirse, es ocupado por nuevos y diversificados agentes que intervienen en el ecosistema de producción y acceso:

- Locales de fotocopiado, gestionados por pequeños y medianos comerciantes o por los propios estudiantes en centros de fotocopiado estudiantil. En estos,los docentes y estudiantes seleccionan y ponen a disposición los materiales para su fotocopiado.

- Comunidades de intercambio online de las que participan docentes y estudiantes.

- El Entorno Virtual de Aprendizaje EVA/UdelaR. Es una de las plataformas Moodle con más usuarios en el mundo, por ella circula gran cantidad de material de estudio. Los docentes comienzan a elaborar y distribuir recursos educativos para sus estudiantes. Algunos de ellos pueden considerarse REA, o cercanos a REA. El EVA también es utilizado para disponibilizar copias no autorizadas de textos, convirtiéndose en lo que se denomina "biblioteca oculta".

- El Portal Timbó de acceso a publicaciones científicas, primera iniciativa estatal de acceso digital a través del pago de licencias a grandes editoriales y revistas arbitradas.

- Revistas y repositorios académicos de Acceso Abierto, como es el caso de Colibrí (Conocimiento Libre Repositorio Institucional) de la Udelar

Frente a este panorama, las editoriales se resisten al nuevo escenario presionando por un combate a la "piratería" de libros. Por el lado del acceso, una gran cantidad de docentes y estudiantes cuentan con una mayor diversidad de materiales a precios más bajos, e incluso gratuitamente. Las bibliotecas, restringidas por el bajo presupuesto y por las limitaciones relacionadas con los contratos editoriales, ven reducida su capacidad de participar como agentes de compras de libros nuevos, o a través de servicios de copias reprográficas o digitales de sus acervos.

Podemos citar el estudio exploratorio realizado por Rodés et al (2012); Rodés y Pérez Casas (2013) como un antecedente que refleja còmo los estudiantes acceden en la práctica a los materiales de estudio. Este estudio, supuso la recolección y análisis de datos acerca de las necesidades específicas de los libros de texto en América Latina en el marco de una iniciativa de libros de texto abiertos (LATIn, 2015) que involucró la participación de nueve universidades. El estudio se desarrolló en base a una consulta

\footnotetext{
${ }^{1}$ El número de ingresos a la UdelaR aumentó un 58,7\% entre 2000 y 2012 (Anuario estadístico del Ministerio de Educación y Cultura -2013)
} 
abierta en línea que, en el caso de la Udelar, obtuvo 771 respuestas de estudiantes de diferentes facultades.

Si bien la muestra obtenida en este estudio no es estadísticamente representativa, en la medida en que es amplia y participativa, permite elaborar perfiles generales. A continuación destacamos algunos resultados:

- Algo más del 40\% de los estudiantes declaran que no pueden acceder a textos obligatorios.

- Un 20\% declara que "no compra libros".

- El 57\% afirma que la compra de libros de textos universitarios le insume una parte significativa de su presupuesto.

- El 66\% declara que utiliza "fotocopias en papel de capítulos sueltos de libros de texto".

- El 43\% declara que utilizaron "libros digitales bajados de la web, sin las licencias correspondientes".

- El 56\% de los estudiantes que utiliza libros digitales prefiere "imprimirlos en papel para leerlos".

En conclusión, los estudiantes apelan habitualmente a estrategias diversas para acceder a los materiales de estudio, con una preferencia por el formato en papel. Incluso entre quienes utilizan libros digitales, el 56\% imprime los archivos digitales, ya que aún es baja la adopción de dispositivos de lectura portátil, como e-readers y tabletas.

Cuando se indaga sobre sus actitudes hacia los libros de texto digitales, las tres características más valoradas por los estudiantes universitarios son: que los libros estén disponibles en Internet, que sean gratis y que se puedan imprimir (muy por detrás quedaron consideraciones sobre compatibilidad con dispositivos, buen diseño $o$ inclusión de materiales multimedia).

En suma, el estudio retrata a una población de estudiantes universitarios con dificultades para acceder a los libros de sus carreras y que, a través de medios diversos, manejan una serie de estrategias para acceder a los materiales de estudio.

En el marco del estudio mencionado se realizó también una consulta abierta dirigida a los docentes, con las mismas características del caso de la consulta a estudiantes. En este caso Rodés y Pérez (2014) relevan que los docentes, ante la pregunta de si "han publicado algún libro de texto en los últimos tres años", el 77\% de todos los docentes de todas las universidades estudiadas responde negativamente. En el caso de Udelar los que responden afirmativamente muestran una proporción que es muy levemente superior al promedio, con registro del $26 \%$, frente al porcentaje general, que se sitúa en el $23 \%$.

Sin embargo, ante la consulta de si "durante los últimos tres años ha generado material educativo inédito que cree pueda ser interesante publicar en un libro de texto colaborativo", el 69\% de todos los consultados responden afirmativamente.

Podemos complementar estos datos con una observación que consta en el VII Censo de Estudiantes de la Udelar, según el cual el 94,9 \% de los estudiantes utiliza la plataforma EVA principalmente para descargar archivos relacionados con las asignaturas de sus carreras. Estos perfiles dan cuenta de un potencial de publicación de materiales de estudio adaptados cultural y pedagógicamente al contexto de utilización que no está siendo contemplado. 


\section{CBIE-LACLO 2015}

Anais dos Workshops do IV Congresso Brasileiro de Informática na Educação (CBIE 2015)

Tampoco existe un análisis sistemático que describa el funcionamiento del mercado editorial de libros técnicos y científicos. En 2009 el Conglomerado Editorial realizó estudios para un "Plan de refuerzo de la competitividad" para el mercado editorial uruguayo en su conjunto. De acuerdo con los mencionados estudios (DICREA - DNC - MEC, 2009), encontramos un sector editorial reducido y orientado casi en su totalidad al mercado interno. El Estado es un actor de importancia para el sector. Por un lado, actúa como comprador, sobre todo en el segmento de libros para centros de estudio y bibliotecas. Pero también actúa para incentivar la actividad estableciendo subsidios al sector mediante exoneraciones impositivas. La importación y la exportación de libros no están gravadas, y la venta de libros y materiales educativos al público está exonerada de IVA.

El informe del Conglomerado Editorial destaca la escasa piratería en comparación con otros países, como una fortaleza del sector editorial uruguayo. Sin embargo, también está presente la queja tradicional del sector editorial acerca de la falta de respeto de la ley de derecho de autor. Particularmente, se menciona la incidencia negativa del fotocopiado en el sector que nos interesa: el de libros universitarios.

En cuanto al acceso, el informe destaca que Uruguay cuenta con una elevada cantidad de librerías por habitante, aunque la mayoría se encuentran concentradas en Montevideo. En el interior del país existen solo en algunas de las ciudades más grandes.

Por otra parte, Uruguay tiene casi cuatrocientas bibliotecas públicas, privadas y municipales, según el Censo de Bibliotecas realizado en 2007 por la Dirección de Cultura del Ministerio de Educación y Cultura para la Biblioteca Nacional. Se destaca la cantidad de bibliotecas municipales (más de ciento treinta), seguidas por las bibliotecas públicas dependientes de la Administración Central (casi cien). Las bibliotecas privadas con acceso público son aproximadamente una centena. Un dato interesante es que hay muchas más bibliotecas en el interior del país que en Montevideo, lo que contrarresta la menor cantidad de librerías. Más de la mitad del presupuesto de las bibliotecas es financiado con fondos públicos.

En lo que refiere específicamente a las bibliotecas universitarias, encontramos que han recorrido un largo camino para salir de un modelo descentralizado que las mantenía aisladas entre sí, hacia una estructura concertada y que persigue objetivos comunes (Madrid, 2010). Constatamos asimismo que la información referente a su rol y funcionamiento actual es escasa y dispersa.

Finalmente, para completar el panorama de acceso a materiales educativos, debemos mencionar las acciones de la UdelaR en la implementación del Programa de Entornos Virtuales de Aprendizaje (ProEVA) y del Repositorio de Acceso Abierto a la Información Científica (Colibrí).

Los más de 2000 cursos semi-presenciales que aloja el EVA integran miles de contenidos educativos. Todos estos contenidos educativos constituyen un potencial a ser compartido a través de la apertura de los mismos, facilitando el acceso a fuentes relevantes de conocimiento. La Udelar ha tomado posición en tal sentido a través de la Resolución Nro 17 del 19/02/2013 de su Consejo Directivo Central.

Asimismo, el Consejo Directivo Central, en sesión del 25/06/2013, dispuso crear el Repositorio Abierto de Publicaciones de la UdelaR como repositorio de todas las 
CBIE-LACLO 2015

Anais dos Workshops do IV Congresso Brasileiro de Informática na Educação (CBIE 2015)

publicaciones producidas por docentes junto con las tesis de grado y posgrado de la Udelar.

\section{Conclusiones y trabajos futuros}

Del presente estado del arte y relevamiento de los principales debates en torno al acceso a los materiales de estudio universitarios en Uruguay se desprenden la falta de análisis específicos que, de modo exhaustivo, den cuenta de la situación actual y orienten políticas educativas en el futuro, en especial aquellas vinculadas al desarrollo de los Recursos Educativos Abiertos como política institucional de publicación de contenidos educativos.

Ante la inexistencia de relevamientos específicos, desde el Núcleo Interdisciplinario sobre Recursos Educativos Abiertos se encuentra en curso una investigación que, se espera, contribuya al conocimiento del ecosistema de creación, circulación y acceso de materiales educativos.

Este estudio contribuirá, por un lado, a dar cuenta de las características de la estructura editorial institucional y la definición de líneas de mejora, permitiendo el conocimiento de la oferta editorial en la Educación Superior.

Estudiar lo que denominamos "ecosistema de creación, circulación y acceso de materiales educativos" implica una mirada amplia que se enfoca en la articulación entre las opciones del mercado, las políticas públicas, el comportamiento y preferencias de los usuarios y la existencia de canales alternativos que no son reconocidos como legítimos en la normativa que regula la propiedad intelectual.

Este estudio permitirá conocer el ecosistema de creación, circulación y acceso a literatura académica y técnica y comprender las causas de las tensiones existentes en el escenario actual.

Se entiende que esta investigación favorecerá el desarrollo y construcción de políticas públicas nacionales sobre acceso a materiales de estudio basadas en conocimiento empírico, y favorecerá la adopción de las políticas de REA como modelo de publicación sustentable.

\section{Referencias}

Udelar (2012). "VII Censo de Estudiantes Universitarios de Grado (2012). Principales características de los estudiantes de grado de la Universidad de la República en 2012”. Dir. Gral. de Planeamiento. Universidad de la República.

BOAI (2002). "Iniciativa de Budapest para el Acceso Abierto". Recuperado el 22 de agosto de 2015: http://www.budapestopenaccessinitiative.org/translations/spanishtranslation

Boley, H. y Chang, E. (2007). "Digital Ecosystems: Principles and Semantics. Inaugural IEEE International Conference on Digital Ecosystems and Technologies". Cairns, Australia. February 2007. NRC 48813.

Busaniche, B. (2013). "Propiedad Intelectual y Derechos Humanos. Tensiones existentes entre la Ley 11.723 y el marco constitucional de los Derechos Culturales 


\section{CBIE-LACLO 2015}

Anais dos Workshops do IV Congresso Brasileiro de Informática na Educação (CBIE 2015)

en Argentina". Tesis de Maestría. FLACSO. Recuperado el 22 de agosto de 2015 de: http://www.vialibre.org.ar/wpcontent/uploads/2014/07/Tesis.final_Beatriz.Busaniche.pdf

Chang, E. y West, M. (2006). "Digital ecosystems a next generation of the collaborative environment". Proceedings of the 8th International Conference on Information Integration and Web-based Applications \& Services (iiWAS2006), Gabriele Kotsis, David Taniar, Eric Pardede, Ismail Khalil Ibrahim (eds), Östereichische. Computer Gesellschaft, ISBN 3-85403-214-5, books@ocg.at, BAND: 214

Craveiro, G., Machado, J. y Ortellado, P. (2008). "O mercado de livros técnicos e científicos no Brasil: subsídio público e acesso ao conhecimento". Grupo de Pesquisa em Políticas Públicas para o Acesso à Informação, Universidade de São Paulo. Recuperado el 22 de agosto de 2015 de: http://www.gpopai.usp.br/relatoriolivros.pdf

Dias Sobrinho, J. (2008). "Calidad, pertinencia y responsabilidad social de la universidad latinoamericana y caribeña”. En Didriksson, Axel; Gazzola, Ana Lúcia, ed., \& Didriksson, Axel, ed. (2008). Tendencias de la educación superior en América Latina y el Caribe (IESALC-UNESCO.). Caracas: Instituto Internacional de la UNESCO para la Educación Superior en América Latina y el Caribe.

DICREA - DNC - MEC (2009). "Hacia la cuenta satélite de cultura en Uruguay". Recuperado el 22 de agosto de 2015 de: http://cultura.mec.gub.uy/innovaportal/file/38210/1/cuenta_satelite_web.pdf

DICREA - DNC - MEC (2009). "Conglomerado Editorial. Plan de refuerzo de la competitividad". Recuperado el 22 de agosto de 2015 de:

http://www.cultura.mec.gub.uy/innovaportal/file/5535/1/conglomerado_editorial_pla n refuerzo de la competitividad.pdf

Didriksson, A., Gazzola, A. L., ed. y Didriksson, A., ed. (2008). "Tendencias de la educación superior en América Latina y el Caribe". IESALC-UNESCO. Caracas: Instituto Internacional de la UNESCO para la Educación Superior en América Latina y el Caribe.

El País (2013). "Estudiantes juntaron 10 mil firmas a favor de su campaña por fotocopias". Recuperado el 22 de agosto de 2014 de:

http://www.elpais.com.uy/informacion/estudiantes-juntaron-firmas-favorcampana.html

FEUU (2013). "Declaración a la opinión pública por materiales de estudio". Recuperado el 25 de mayo de 2014 de: https://www.facebook.com/notes/federacionestudiantes-universitarios-uruguay/declaraci $\% \mathrm{C} 3 \% \mathrm{~B} 3 n-\mathrm{a}-1 \mathrm{a}-$ opini $\% \mathrm{C} 3 \% \mathrm{~B} 3 \mathrm{n}-\mathrm{p}$ \%C3\%BAblica-por-materiales-de-estudio/10152045270816719

Geertz, C. (1973). "The interpretation of cultures: selected essays". Basic Books.

Gemetto, J. (2013). “Acceso a la cultura y derechos de autor en Uruguay: \#noal218, una victoria de la sociedad civil". En Digital Rights LAC, numero 3, 09/2013. Recuperado el 22 de agosto de 2015 de: http://www.digitalrightslac.net/es/acceso-ala-cultura-y-derechos-de-autor-en-uruguay-noal218-una-victoria-de-la-sociedad-civil

Glaser, B. G., y Strauss, A. L. (1967). "The discovery of grounded theory: strategies for qualitative research". Aldine Pub. Co. 


\section{CBIE-LACLO 2015}

Anais dos Workshops do IV Congresso Brasileiro de Informática na Educação (CBIE 2015)

Instituto Nacional de Estadísticas (2013). "Uruguay en Cifras, Educación y Cultura". Recuperado el 22 de agosto de 2015 de: http://www.ine.gub.uy/biblioteca/uruguayencifras2013/capitulos/Educaci\%C3\%B3n $\% 20 \mathrm{y} \% 20$ Cultura.pdf

IESALC (2008). "Declaración de la Conferencia Regional de Educación Superior en América Latina y el Caribe - CRES 2008." Presentado en la Conferencia Regional de Educación Superior en América Latina y el Caribe - CRES 2008, Cartagena de Indias, Colombia: IESALC Instituto Internacional de la UNESCO para la Educación Superior en América Latina y el Caribe.

Madrid, I. (2010). "La integración en un Sistema de Bibliotecas de la Universidad de la República: Una mirada desde las políticas educativas universitarias". Informatio (14/16): 7-23, 2009-2011, ISSN 0797-1435. Recuperado el 22 de agosto de 2015 de: http://www.eubca.edu.uy/sites/default/files/text/informatio/14 16/5 madrid_isabel.p df

Montevideo Portal (2013, noviembre). “CED presentó proyecto a Astori. Prácticas preprofesionales". Recuperado el 22 de agosto de 2015 de: http://www.montevideo.com.uy/notnoticias_218362_1.html

Noronha F. y Malcolm, J. (2010). “Acceso al conocimiento: Una guía para todos”. Consumers International Regional Office for Asia Pacific and the Middle East. Recuperado el 22 de agosto de 2015 de: http://www.consumersinternational.org/media/457953/a2k-spanish.pdf

Rodés, V., Pérez Casas, A., Ochoa, X. y Frango, I. (2012): "Percepciones, actitudes y prácticas respecto a los libros de texto, digitales y en formatos abiertos por parte de estudiantes de universidades de América Latina" I Workshop em Recursos Educacionais Abertos. Congresso Brasileiro de Informática da Educação, Río de Janeiro, Brasil. Recuperado el 22 de agosto de 2015 de: http://www.brie.org/pub/index.php/wcbie/article/view/1893

Rodés, V., Pérez Casas, A. y Podetti, M. (2014). "Percepciones, actitudes y prácticas respecto a los libros de texto y al uso de libros digitales en formatos abiertos". II Jornada de Investigación en Educación Superior. Montevideo, Uruguay.

Shaver, L. (2008) "Defining and Measuring A2K: A Blueprint for an Index of Access to Knowledge”. Indiana University Robert H. McKinney School of Law, I/S: A Journal of Law and Policy for the Information Society 4, no. 2: 235-269. Recuperado el 22 de agosto de 2015 de: http://papers.ssrn.com/sol3/papers.cfm?abstract id=1021065

Shaver, L. (2014) “Copyright and Inequality”, Washington University Law Review 117 (2014), Indiana University Robert H. McKinney School of Law Research Paper No. 2014-3. Recuperado el 22 de agosto de 2015 de: http://papers.ssrn.com/sol3/papers.cfm?abstract id $=2398373$

Sumar Uy (2013). "Informes de las Conferencias Ciudadanas Sumar”. Recuperado el 22 de agosto de 2015 de: http://www.sumar.uy/?page_id=9272 